The Bretherton Problem in Elastic-Walled Channels: Finite Reynolds Number Effects

Heil, Matthias

2001

MIMS EPrint: 2009.78

Manchester Institute for Mathematical Sciences

School of Mathematics

The University of Manchester

\footnotetext{
Reports available from: http://eprints.maths.manchester.ac.uk/

And by contacting: The MIMS Secretary

School of Mathematics

The University of Manchester

Manchester, M13 9PL, UK
} 


\title{
THE BRETHERTON PROBLEM IN ELASTIC- WALLED CHANNELS: FINITE REYNOLDS NUMBER EFFECTS
}

\author{
Matthias Heil \\ Department of Mathematics, University of Manchester \\ M.Heil@maths.man.ac.uk
}

\begin{abstract}
This paper investigates the effect of fluid inertia on the propagation of an air finger into a channel with elastic walls, a problem which can be regarded as a generalisation of the classical Bretherton problem. The study is motivated by the physiological problem of pulmonary airway reopening. Numerical results show that fluid inertia plays a surprisingly important role in this problem: Even for relatively modest ratios of Reynolds and Capillary numbers $(R e / C a \approx 5-10)$, the pressure required to drive the air finger at a given speed increases significantly compared to the zero Reynolds number case. Inertial effects are also shown to be responsible for a noticeable change in the wall deformation ahead of the bubble tip. This is analysed by a Karman-Pohlhausen approximation which yields a linear ODE, the eigenvalues of which determine the wavelength and decay rate of the oscillatory wall displacement field in this region.
\end{abstract}

Keywords: Bretherton problem, fluid-structure interaction, pulmonary airway reopening

\section{INTRODUCTION}

The Bretherton problem (the propagation of an air finger into a rigidwalled fluid-filled channel; Bretherton 1961) is a classical free-surface flow problem. Gaver et al. (1996) proposed a generalisation of this problem as a model of pulmonary airway reopening: They considered the steady propagation of an air finger into a two-dimensional, fluid-filled channel whose flexible walls are represented by membranes under tension. The walls are supported on an elastic foundation (of stiffness $K^{*}$ ) which represents the tethering provided by the lung tissue surrounding the occluded and collapsed airway. The model problem is illustrated in 
Fig. 1. Gaver et al. (1996) neglected fluid inertia, an approximation which was justified by the (assumed) small Reynolds numbers in airway reopening and in benchtop experiments by, e.g., Gaver et al. (1990) and Yap et al. (1994). The Stokes equations were solved by a boundary element method which incorporated an Eulerian description of the wall mechanics. Gaver et al. (1996) provided a detailed analysis of this system and determined the non-dimensional propagation speed of the bubble tip (expressed in terms of the capillary number $C a=U \mu / \gamma^{*}$ ) as a function of the applied bubble pressure $p_{b}$.

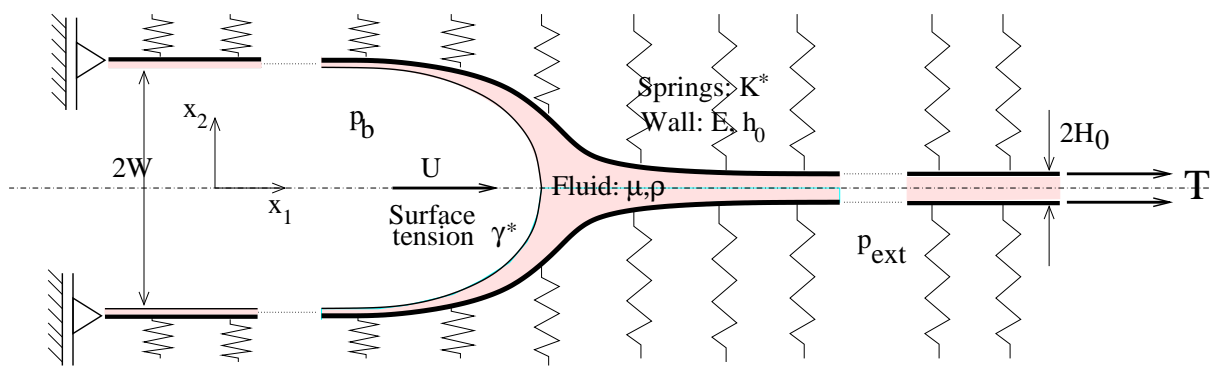

Figure 1. Sketch of the Bretherton problem with elastic walls: An air finger propagates into a liquid-filled two-dimensional channel with elastic walls.

\section{FINITE REYNOLDS NUMBER EFFECTS}

Heil (2000) re-investigated the problem and provided several improvements to the original model: (i) Gaver et al.'s (1996) Eulerian wall model which required a number of ad-hoc assumptions in the implementation of the no-slip condition at the fluid-wall interface was replaced by a Lagrangian wall model. This allowed a fully self-consistent description of the fluid-structure interaction and the inclusion of bending stiffness; (ii) the solution of the fluid equations was achieved by a Finite Element discretisation of the free-surface Navier-Stokes equations which allowed the inclusion of fluid inertia into the problem.

Briefly, in Heil's (2000) model, the wall is regarded as a beam of thickness $h_{0}$ which is subject to a large axial tension $T=\sigma_{0} h_{0}$. In a frame moving with velocity of the bubble tip, the wall deformation is described by the principle of virtual displacements

$$
\begin{gathered}
\int_{-\infty}^{\infty}\left[\left(\sigma_{0}+\quad e\right) \delta e+\frac{1}{12}\left(\frac{h_{0}}{H_{0}}\right)^{2} \kappa \delta \kappa-\left(\frac{H_{0}}{h_{0}}\right)\left(-K v^{2}\left(1+v_{, \zeta}^{1}\right) \delta v^{2}+\right.\right. \\
\left.\left.+\quad \mathbf{f} \cdot \delta \mathbf{R}_{w} \sqrt{\left(1+v_{, \zeta}^{1}\right)^{2}+\left(v_{, \zeta}^{2}\right)^{2}}\right)\right] d \zeta=0
\end{gathered}
$$


where $\mathbf{R}_{w}(\zeta)$ is the vector to a material point on the deformed wall (parametrised in terms of the Lagrangian travelling wave coordinate $\zeta$ ), the $v^{i}$ are the components of the displacement vector and $e$ and $\kappa$ represent the wall's incremental strain and change of curvature, respectively. $\mathbf{f}$ is the traction (non-dimensionalised by the wall's elastic modulus $E$ ) acting on the wall and $K=K^{*} H_{0} / E$.

The flow is described by the free-surface Navier-Stokes equations

$$
\begin{gathered}
\text { Re } u_{j} \frac{\partial u_{i}}{\partial x_{j}}=-\frac{\partial p}{\partial x_{i}}+\frac{\partial}{\partial x_{j}}\left(\frac{\partial u_{i}}{\partial x_{j}}+\frac{\partial u_{j}}{\partial x_{i}}\right) \\
\frac{\partial u_{i}}{\partial x_{i}}=0
\end{gathered}
$$

together with the interfacial boundary conditions

$$
\left.\begin{array}{c}
\mathbf{u} \cdot \mathbf{n}=0 \\
-p n_{i}+\left(\frac{\partial u_{i}}{\partial x_{j}}+\frac{\partial u_{j}}{\partial x_{i}}\right) n_{j}+\frac{1}{C a} \kappa_{f} n_{i}=-p_{b} n_{i}
\end{array}\right\} \quad \text { on the free surface, }
$$

where the velocities were scaled with the bubble velocity $U$ and the viscous pressure scale was used. $\kappa_{f}$ is the non-dimensional curvature of the air-liquid interface and $R e=U \rho H_{0} / \mu$. The fluid and solid domains are coupled via the no-slip condition

$$
\mathbf{u}=-\left(1+v_{, \zeta}^{1}, v_{, \zeta}^{2}\right) \quad \text { on the wall }
$$

and by the traction that the fluid exerts on the wall. Taking the different stress non-dimensionalisations in the fluid and solid domains into account, the traction on the wall is given by

$$
f_{i}=C a \gamma\left(p n_{i}-\left(\frac{\partial u_{i}}{\partial x_{j}}+\frac{\partial u_{j}}{\partial x_{i}}\right) n_{j}\right) .
$$

Here the non-dimensional surface tension $\gamma=\gamma^{*} /\left(E H_{0}\right)$ represents the ratio of the fluid surface tension to the wall's extensional stiffness.

Heil (2000) showed that even moderate fluid inertia significantly increases the bubble pressure $p_{b}$ required to drive the bubble at a given speed (capillary number), as shown in Fig. 2. Note that the ratio of capillary and Reynolds numbers (both of which scale linearly with the bubble velocity) is kept constant along the various curves in this figure, to ensure that the curves represent physically realisable parameter variations. The corresponding plots of the fluid domain in Fig. 3 show that fluid inertia leads to a significant change in the wall displacement field ahead of the bubble tip: At finite Reynolds number, the wall develops an oscillatory deformation pattern whose wavelength, decay rate and amplitude vary strongly with the Reynolds number. 


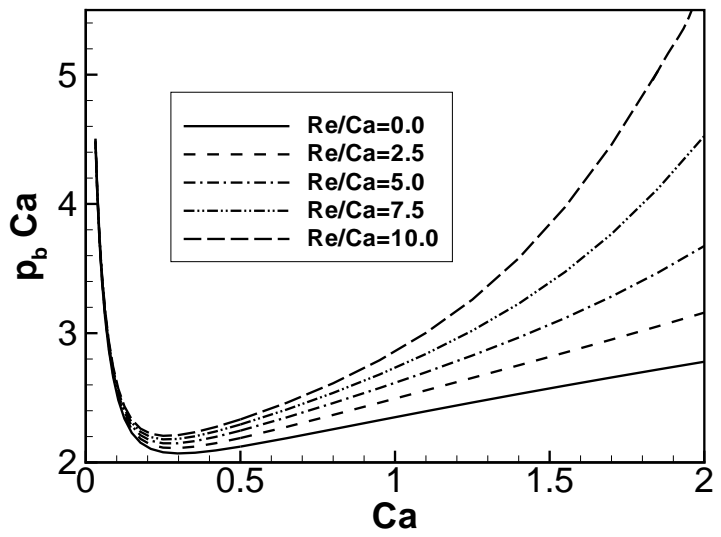

Figure 2. The bubble pressure (on the capillary pressure scale) versus the nondimensional bubble speed $C a=U \mu / \gamma^{*}$ for various values of $R e / C a$.

\section{AN ANALYTICAL MODEL}

We will now develop an analytical model to analyse the changes to the wall displacement field at finite Reynolds number. The wall displacement field ahead of the bubble tip approaches the system's decaying eigenfunctions as $x_{1} \rightarrow \infty$. At zero Reynolds number, the eigenfunction can be determined from a lubrication theory analysis (Gaver et al. 1996). To extend the analysis to finite Reynolds number, we note that Fig. 3 shows that in the region ahead of the bubble tip (i) the pressure remains approximately uniform over the width of the channel ${ }^{1}$ and (ii) the wall slope remains moderate (note the different scales for the $x_{1}$ and $x_{2}$-axes).

Motivated by these observations, we consider the case in which the small wall slope, $d v^{2} / d \zeta=\mathcal{O}(\epsilon) \ll 1$ implies small transverse fluid velocities such that $u_{2} / u_{1}=\mathcal{O}(\epsilon)$, but assume that fluid inertia is so large that $\epsilon R e=\mathcal{O}(1)$. Inserting these scalings into the momentum equation yields the leading order contributions

$$
R e\left(u_{1} \frac{\partial u_{1}}{\partial x_{1}}+u_{2} \frac{\partial u_{1}}{\partial x_{2}}\right)=-\frac{\partial p}{\partial x_{1}}+\frac{\partial^{2} u_{1}}{\partial x_{2}^{2}}
$$

and $\partial p / \partial x_{2}=0$. In view of the small wall deflections, we linearise the wall equations (which represent the Euler-Lagrange equations of the

${ }^{1}$ At sufficiently large Reynolds number, a local pressure rise towards the bubble tip can be detected; see the inset in Fig. 3. This pressure rise reduces the curvature of the air-liquid interface near the bubble tip and can even cause it to 'bulge out'. 


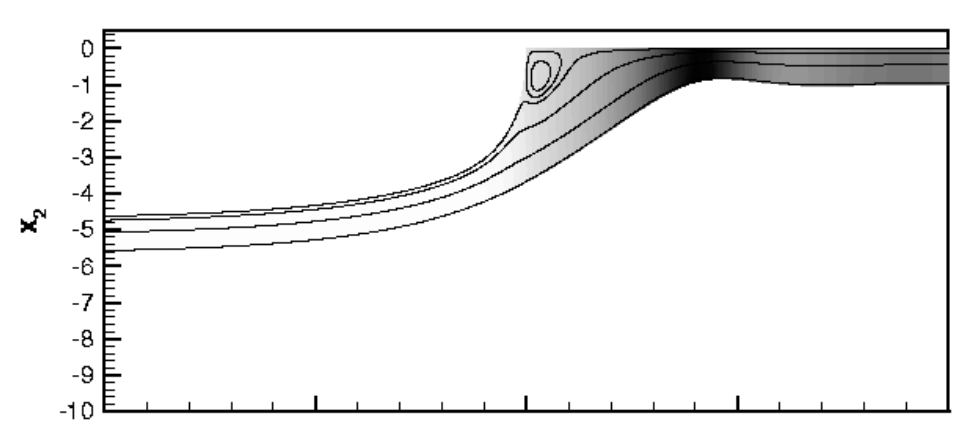

$\mathrm{Re} / \mathrm{Ca}=10$

$\mathrm{Ca}=0.5$
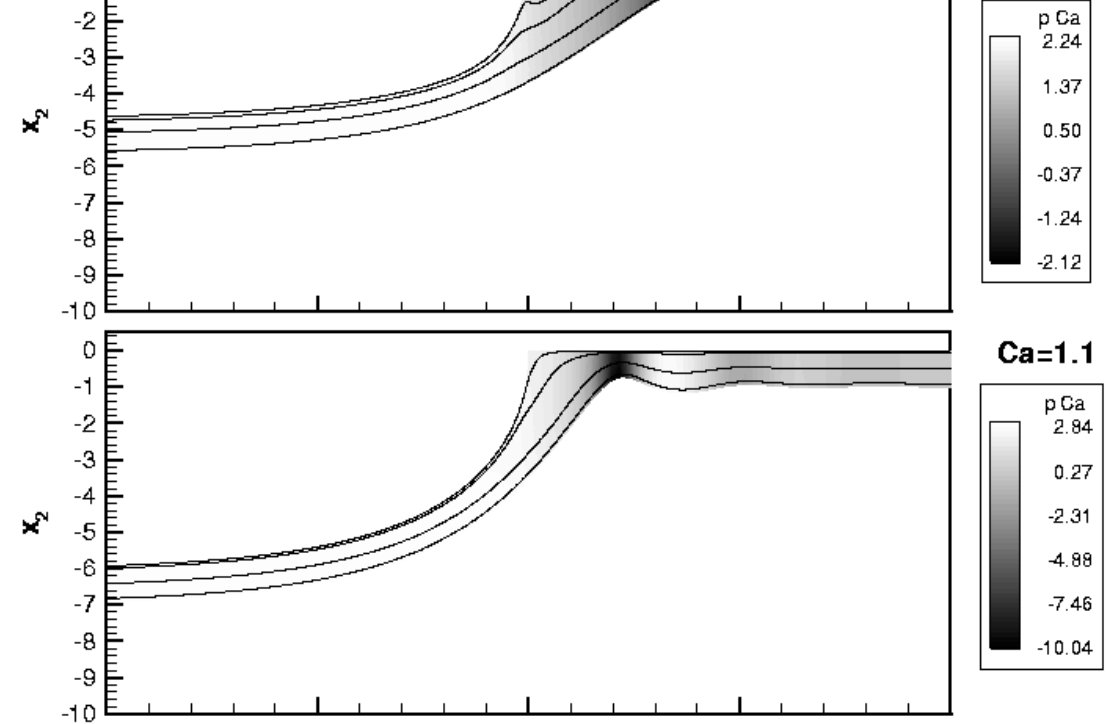

$\mathrm{Ca}=1.1$
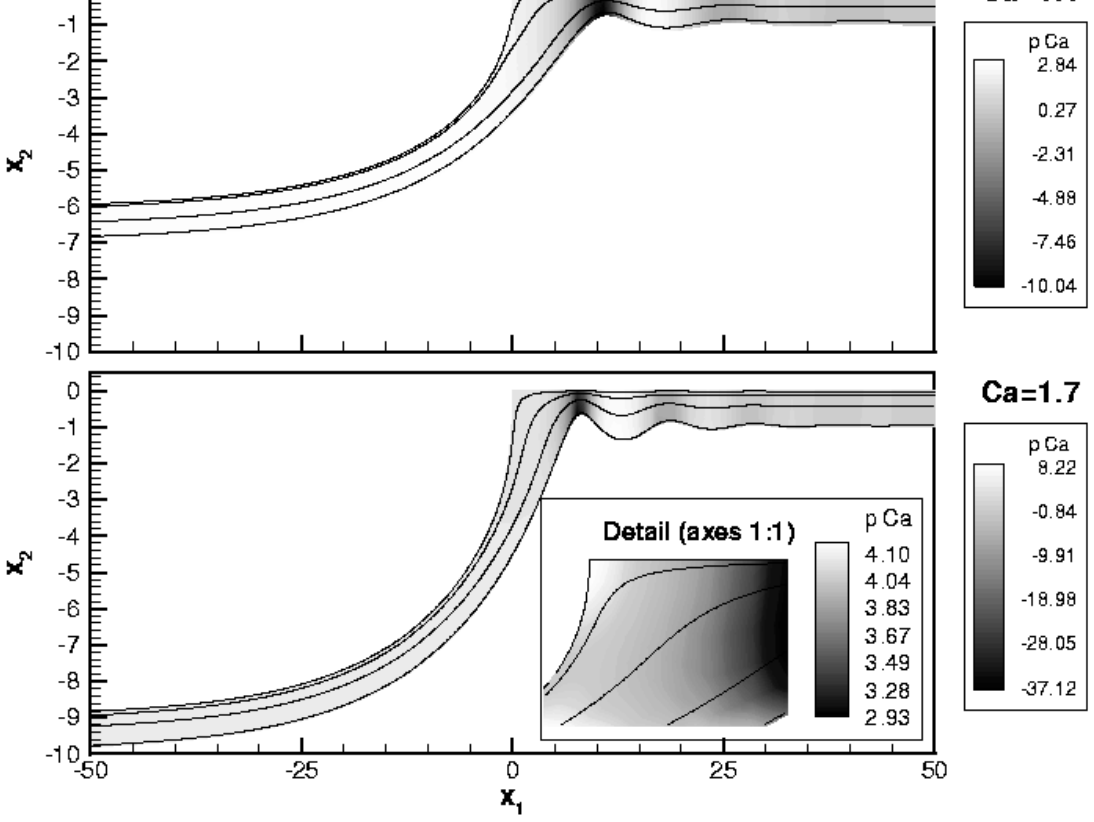

$\mathrm{Ca}=1.7$

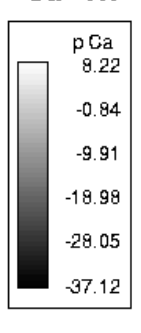

Figure 3. Streamlines and pressure contours (on the capillary pressure scale) for various $C a$ and for $R e / C a=10$. Only the lower half of the channel is shown.

variational principle (1)) to obtain the following equation for the channel half-width $w\left(x_{1}\right)$

$$
p=\frac{1}{C a}\left(\Gamma(w-1)-\eta w^{\prime \prime}+\frac{1}{12 \gamma}\left(\frac{h_{0}}{H_{0}}\right)^{3} w^{i v}\right) .
$$

Here $\eta=T / \gamma^{*}$ and $\Gamma=K^{*} H_{0}^{2} / \gamma^{*}$ represent Gaver et al.'s (1996) nondimensionalisations for the wall tension and the stiffness of the elastic foundation, respectively. The incremental wall strain $e$ tends to zero as 
$x_{1} \rightarrow \infty$, therefore the velocity boundary condition (2) becomes $u_{1}=-1$ and $u_{2}=-w^{\prime}$ at $x_{2}=w$. Following Christodoulou \& Scriven (1989), we use the Karman-Pohlhausen method and approximate the velocity distribution by

$$
u_{1}\left(x_{1}, x_{2}\right)=-1+\frac{3}{2} \frac{w\left(x_{1}\right)-1}{w\left(x_{1}\right)}\left(1-\left(\frac{x_{2}}{w\left(x_{1}\right)}\right)^{2}\right)
$$

and

$$
u_{2}\left(x_{1}, x_{2}\right)=\frac{x_{2}}{w\left(x_{1}\right)} w^{\prime}\left(x_{1}\right) u_{1}\left(x_{1}, x_{2}\right)
$$

and substitute (4) - (6) into (3). Integrating the momentum equation (3) over the channel width yields a nonlinear equation for $w\left(x_{1}\right)$. We linearise this equation with respect to $\omega\left(x_{1}\right)=w\left(x_{1}\right)-1=\mathcal{O}(\epsilon)$ to obtain the homogeneous ODE

$$
\operatorname{Re} C a \omega^{\prime}=\Gamma \omega^{\prime}-\eta \omega^{\prime \prime \prime}+\frac{1}{12 \gamma}\left(\frac{h_{0}}{H_{0}}\right)^{3} \omega^{v}+3 C a \omega,
$$

which governs the wall deformation as $x_{1} \rightarrow \infty$ and $\omega \rightarrow 0$. Its solution is of the form

$$
\omega \sim \exp \left(\Lambda x_{1}\right)=\exp \left((-\lambda+i \alpha) x_{1}\right)
$$

where only three of the five roots, $\Lambda$, of the characteristic polynomial

$$
\frac{1}{12 \gamma}\left(\frac{h_{0}}{H_{0}}\right)^{3} \Lambda^{5}-\eta \Lambda^{3}+(\Gamma-R e C a) \Lambda+3 C a=0
$$

have negative real part, i.e. are decaying as $x_{1} \rightarrow \infty$. Two of these roots are complex and represent the decaying oscillatory wall displacement field that we wish to analyse. The third negative root is large and purely real and is associated with the short-lengthscale bending deformation. Note that the Reynolds number only appears in the combination Re $C a=U^{2} \rho H_{0} / \sigma$, which is also known as the Weber number, $W e$, and represents the ratio of inertia to surface tension.

We will now investigate how variations of the system's non-dimensional parameters affect the wall displacement field in the presence of fluid inertia. As in Gaver et al. (1996) and Heil (2000), we regard $\eta=100, \Gamma=$ $0.5, h_{0} / H_{0}=5 \times 10^{-4}$ and $\gamma=10^{-7}$ as the reference state.

Fig. 4 shows the variations of the wavenumber $\alpha$ and the decay rate $\lambda$ as a function of the non-dimensional bubble velocity $C a$ for several values of $\mathrm{Re} / \mathrm{Ca}$ : An increase in $\mathrm{Ca}$ increases the wavenumber $\alpha$ and thus shortens the wavelength of the deformation pattern. Fluid inertia can be seen to significantly enhance this effect. The decay rate $\lambda$ increases 

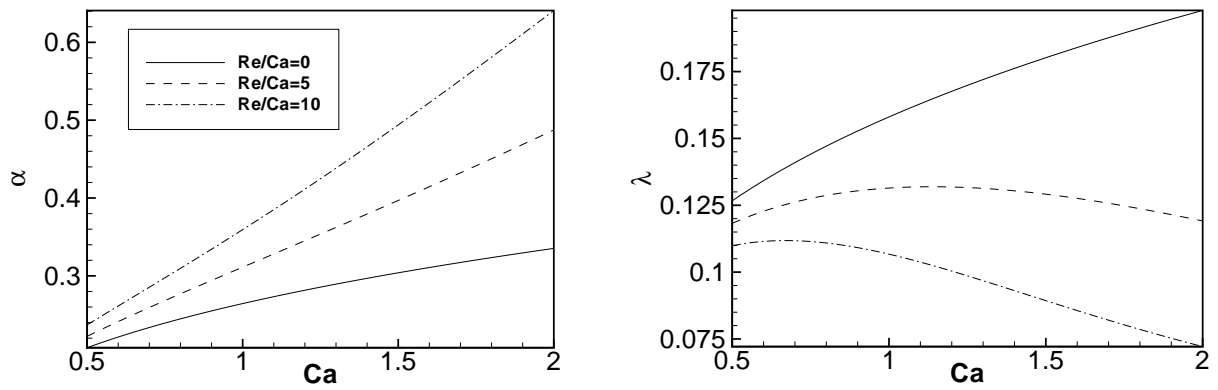

Figure 4. Variation of the wavenumber $\alpha$ and decay rate $\lambda$ with $C a$ for various values of $R e / C a$.
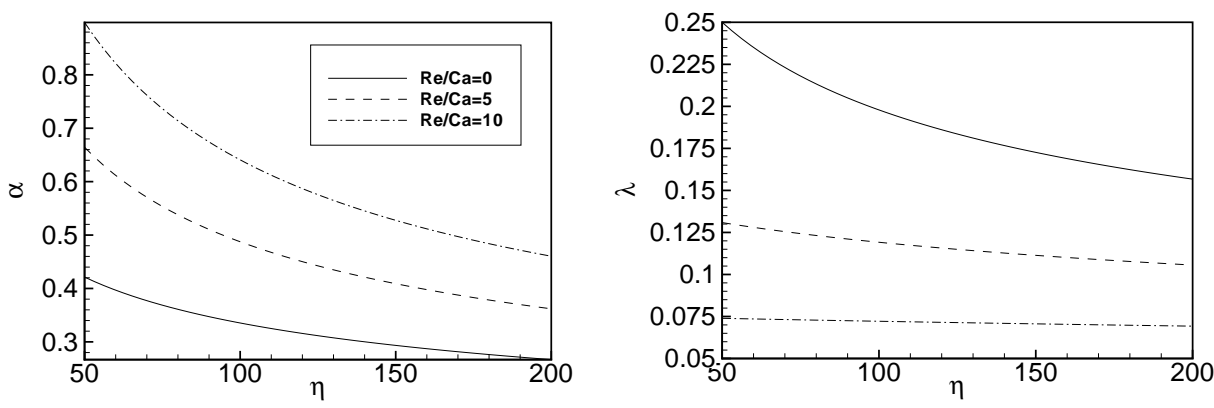

Figure 5. Variation of the wavenumber $\alpha$ and decay rate $\lambda$ with wall tension $\eta$ for various values of $R e / C a$ and for $C a=2$.

with $C a$ when $R e / C a$ is small; this effect is reduced and finally reversed as $\mathrm{Re} / \mathrm{Ca}$ increases.

The changes to the wall displacement field due to variations in the wall tension $\eta$ are illustrated in Fig. 5: An increase in wall tension smoothes out the wavy wall displacement by reducing both the decay rate and the wavenumber. Fluid inertia has a mainly quantitative effect on this behaviour but it is interesting to note that at sufficiently large values of $R e / C a$, the decay rate $\lambda$ becomes nearly independent of the wall tension $\eta$.

Fig. 6 shows that variations in the stiffness of the elastic foundation $\Gamma$ only have a moderate effect on the displacement field ahead of the bubble tip even though they strongly affect the system's overall behaviour (see Heil 2000). It is interesting to note that, according to the linear theory, for a given value of $C a$, changes to $\Gamma$ can be compensated for by a suitable increase in the Weber number $W e$, since $\Gamma$ only appears in the combination $(\Gamma-R e C a)$. 

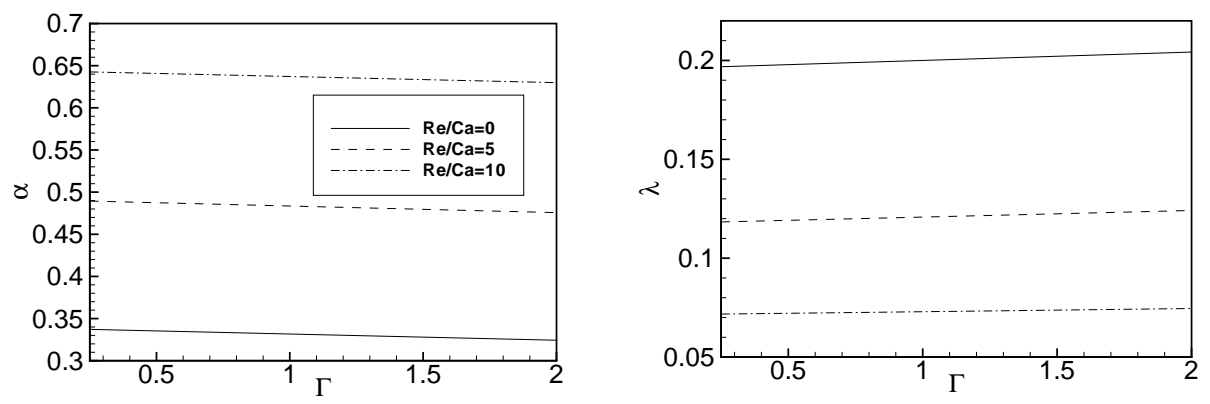

Figure 6. Variation of the wavenumber $\alpha$ and decay rate $\lambda$ with spring stiffness $\Gamma$ for various values of $R e / C a$ and for $C a=2$.

The predictions shown in Figs. $4-6$ are in excellent agreement with the trends observed in the computational parameter studies. This confirms that, as speculated in Heil (2000), the changes to the wall displacement field ahead of the bubble tip are mainly due to the Bernoulli effect.

\section{References}

Bretherton, F. 1961 The motion of long bubbles in tubes. Journal of Fluid Mechanics 10, 166-188.

Christodoulou, K. \& Scriven, L. 1989 The fluid mechanics of slide coating. Journal of Fluid Mechanics 208, 321-354.

Gaver, D., Halpern, D., Jensen, O. \& Grotberg, J. 1996 The steady motion of a semi-infinite bubble through a flexible walled channel. Journal of Fluid Mechanics 319, 25-56.

Gaver, D., SAmSel, R. \& Solway, J. 1990 Effects of surface tension and viscosity on airway reopening. Journal of Applied Physiology 369, 74-85.

Heil, M. 2000 Finite Reynolds number effects in the propagation of an air finger into a liquid-filled flexible-walled channel. Journal of Fluid Mechanics 424, 21-44.

Yap, D., Liebkemann, W., Solway, J. \& Gaver, D. 1994 Influences of parenchymal tethering on the reopening of closed pulmonary airways. Journal of Applied Physiology 76, 2095-2105. 